\title{
OUR FIRST EXPERIENCE WITH MAGNETIC SEPARATION OF PLATELETS FOR ANALYSES OF Platelet microRNA in Patients With Sticky Platelet Syndrome
}

\author{
Vadelova $L^{1}$, Ivankova $J^{1}$, Sokol $J^{1}$, Skerenova $M^{2}$, Zolkova $\mathrm{J}^{1}$, VaZanova $\mathrm{A}^{1}$, \\ SKORNOVA $\mathrm{I}^{1}$, STASKO $\mathrm{J}^{1}$.
}

\begin{abstract}
${ }^{1}$ Department of Hematology and Transfusiology, National Centre of Hemostasis and Thrombosis, Jessenius Faculty of Medicine in Martin, Comenius University in Bratislava, Martin University Hospital, Martin, Slovakia

${ }^{2}$ Divison of Molecular Medicine, Biomedical Center Martin Jessenius Faculty of Medicine in Martin, Comenius University in Bratislava, Martin University Hospital, Martin, Slovakia
\end{abstract}

\section{A bs tract}

Introduction: Sticky platelet syndrome (SPS) is referred to as a platelet hyperaggregability triggered by low concentrations of platelet agonists adenosine diphosphate (ADP) and/or epinephrine (EPI). Platelet aggregation with other inducers (collagen, arachidonic acid, ristocetin, and thrombin) remains within a normal range. MicroRNAs (miRNAs) are small, non-coding RNA molecules that play an important role in post-transcriptional regulation of protein expression. More recently, several studies show that the platelets are an abundant source of miRNAs and that the miRNA expression profiles within platelets correlate with the platelet reactivity.

Aim: The principle objective of this article is to describe the method which we developed for the preparation of the pure platelet samples and report the results of this method. These final pure platelet samples are intended to be the first step for the platelet miRNA testing.

Methods: The blood samples from 50 subjects were examined in the study. Then, the platelet rich plasma (PRP) samples obtained by centrifugation of the patient blood samples were used for our experiments. Subsequently, the erythrocytes and leucocytes remaining in PRP sample were magnetically labelled by CD45 Microbeads and CD235a Microbeads. After incubation the PRP sample passed through the magnetic separation system and the magnetically labelled cells (erythrocytes and leucocytes) were retained within the column of separator. The number of cells in the final PRP samples was measured by the blood cell analyser.

Results and conclusion: We successfully developed and optimized the effective and reproducible method for magnetic separation of platelets, resulting in the leukocyte-depleted and erythrocyte-depleted platelet samples, which can be used for further genetic analyses.

Key words: platelet, magnetic separation, sticky platelet syndrome, platelet miRNA

\section{INTRODUCTION}

\section{Sticky Platelet Syndrome}

Sticky platelet syndrome (SPS) was defined as a platelet disorder with an autosomal-dominant trait, characterized by an increased in vitro platelet aggregation in response to low concentrations of platelet agonists adenosine diphosphate (ADP) and/or epinephrine (EPI). Aggregation with other inducers (collagen, arachidonic acid, ristocetin, and thrombin) remains normal [1]. However, after more than 30 years of research it is necessary to modify this definition. As discussed later, SPS has a multifactorial etiology in which genetic and environmental factors play a role. In addition, this syndrome is characterized by the composite set of laboratory and clinical signs. Therefore, SPS should be considered as a multi-

Corresponding author: Lubica Vadelova; e-mail: vadelova2@uniba.sk

(C) 2019 Lubica Vadelova et al.

This work is licensed under the Creative Commons Attribution-NonCommercial-NoDerivs 4.0 License (https://creativecommons.org/licenses/by-nc-nd/4.0/) 
factorial qualitative platelet disorder characterized by the occurrence of venous or arterial thrombosis, migraine, graft versus host disease (GVHD), or pregnancy complications, in the presence of in vivo permanent enhanced platelet aggregation following low concentrations of ADP and/or EPI. The above-mentioned factors work together in a way that has not yet been clearly described. At present, it can only be assumed that these factors cause changes in platelet glycoprotein membranes or intracellular signaling pathways that are involved in platelet activation and aggregation. These studies, as well as the laboratory heterogeneity of SPS (three different types), point to complex genetics, as it is known in other hemostatic disorders (e.g. von Willebrand's disease) $[3,17]$. It is also important to mention studies that describe platelet hyperaggregability (induced by ADP and EPI natural agonists) associated with a subsequent risk of thrombosis in patients with several chronic diseases such as complex metabolic diseases (diabetes mellitus and arteriosclerosis) and inflammatory diseases (systemic immune diseases) [3,4,5].

In general, we can say that an isolated genetic abnormality that would be responsible for platelet hyperaggregation has not been identified yet [6].

\section{Platelet miRNA}

MiRNAs are small endogenous RNAs consisting of $\sim 22$ nucleotides that post-transcriptionally regulate gene expression by pairing to the 3'untranslated region (3'UTR). MiRNA was discovered accidentally in 1993 by Lee et al. in the research of the timing of the stages of Caenorhabditis elegans nematodes [2,7]. Since that time it has been shown that miRNAs play an essential role in gene regulation in almost every physiologic process, such as metabolism, proliferation, cell growth or differentiation, in all species.

Platelets were the first elements of the hemostatic system that were shown to be regulated by miRNAs. Platelets contain the machinery required for miRNA maturation and function (e.g., Dicer1 and Argonaute2). To date, >500 miRNAs have been characterized in platelets. However, the role of miRNAs in platelets remains poorly understood and few studies identified miRNAs with a specific role in platelet function. MiRNA-223 is the most highly expressed in platelet and seems to play different roles. One of them is the regulation of P2Y12 receptor and, thus, platelet function [8]. Kondkar et al. reported that miRNA-96 seems to regulate VAMP8 expression, vesicular membrane protein involved in platelet secretion, and it also mediates thrombus formation in vivo [9,10]. MiRNA-126 is also among the most highly expressed in platelets [11]. The aggregation response to arachidonic acid was significantly reduced in platelets from mice treated with miRNA-126 inhibitor in whole blood [12]. Therefore, a lot of studies show that platelet can be regulated by miRNAs, opening new and exciting perspectives.

The aim of this article is to describe the method which we developed and optimized for the preparation of the pure platelet samples. We also report our first results of this method. The final pure platelet samples are intended to be the first step for the platelet miRNA testing.

\section{METHODS}

This study includes patients with SPS and healthy controls. Diagnosis of SPS was based on light transmission aggregometry (LTA), using low concentrations of ADP and/or EPI according to Mammen [13] and Bick [14]. The main goal of our work was to prepare the pure platelet samples as an integral part of the platelet miRNA testing.

The leukocyte-depleted platelet concentrate from apheresis was used in our first experiment, because of high number of platelets. The sample of leucocyte-depleted platelet concentrate was centrifuged (400g, $10 \mathrm{~min}$., room temperature). The pellet of platelets on the bottom of test tubes was diluted with MACS Separation Buffer (MiltenyiBiotec, GmbH, Bergisch Gladbach, Germany). To obtain the pure samples of platelets we used magnetic 
separation system (MidiMACS ${ }^{\mathrm{TM}}$ Separator, MiltenyiBiotec, Zürich, Switzerland) (Fig.1). Before the magnetic separation we labelled the cells with superparamagnetic microbeads. We added $100 \mu \mathrm{L}$ of CD45 MicroBeads and 100 $\mu \mathrm{L}$ of CD235a MicroBeads. The CD45 MicroBeads (MiltenyiBiotec GmbH, Bergisch Gladbach, Germany) were used for the positive selection or depletion of leukocytes from the peripheral blood. In addition, the CD235a (glycophorin A) MicroBeads (MiltenyiBiotec MiltenyiBiotec GmbH, Bergisch Gladbach, Germany) were used for the positive selection or depletion of human erythroid cells. After incubation and magnetic labelling the cells were passed through the column which is placed in a strong permanent magnet of the separator. Unlabelled cells passed through the column while magnetically labelled cells were retained within the column. The number of cells in the final platelet samples was determined by the blood cell analyser.

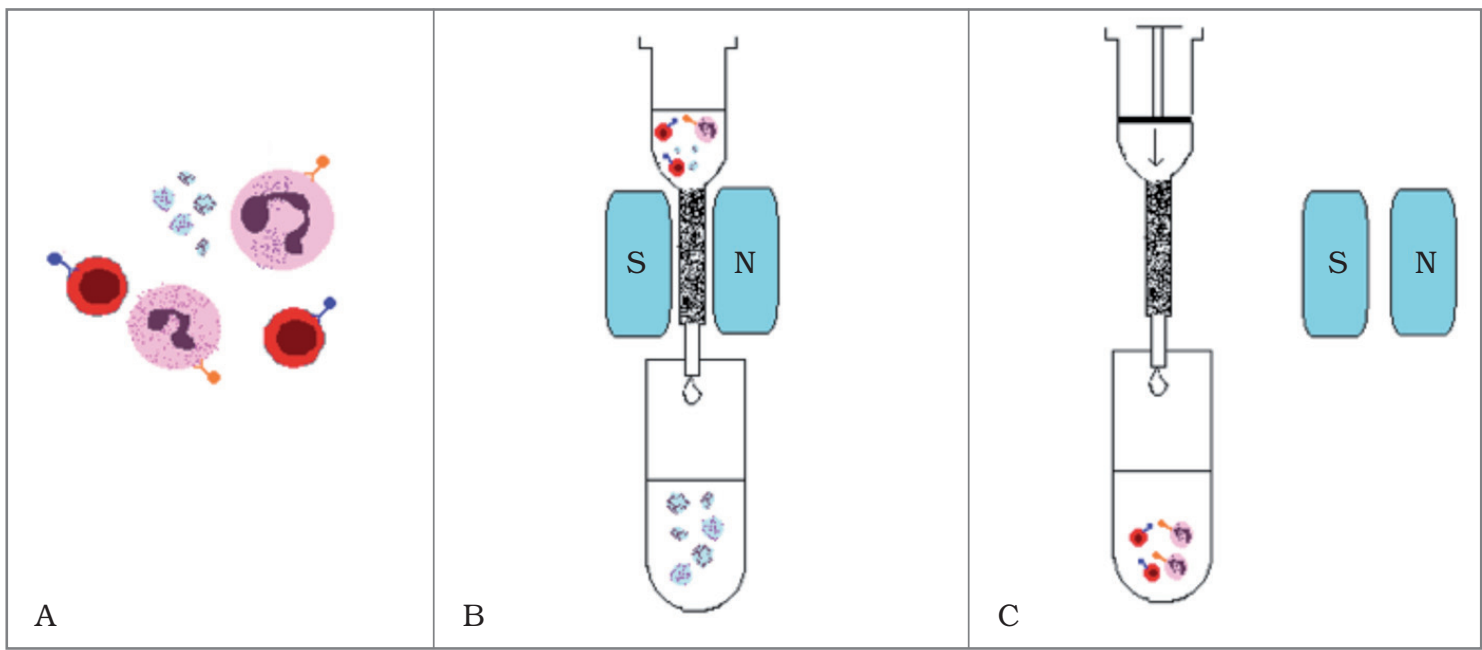

Fig. 1. A: Magnetic labelling of cells with CD45 and CD235a MicroBeads. B: Magnetic separation of platelets by magnetic separation system (MidiMACS ${ }^{\mathrm{TM}}$ Separator, MiltenyiBiotec, Zürich, Switzerland). C: Elution of labelled cell fraction. (available online: https://www.miltenyibiotec. com/_Resources/Persistent/e7a31d4b47c2c1caa28502c65c10a346f512f3bd/30238_Illustration_positive_selection_dir ect_labeling-1170x475.png)

With the aim to improve the results we changed some conditions in our follow-up experiments. The initial blood count was determined in the whole blood sample taken to the EDTA (ethylendiaminotetraacetic acid) tube. Other blood samples were taken to 3.2\% sodium citrate plastic tubes and the samples were centrifuged $\left(200 \mathrm{~g}, 10 \mathrm{~min} ., 37^{\circ} \mathrm{C}\right)$. Using a plastic pipette we transferred the platelet rich plasma (PRP) into the plastic tubes. Then we added prostaglandin E1 (PGE1) into the PRP to prevent the platelet activation according to the amount of PRP. After that we centrifuged the sample again $\left(400 \mathrm{~g}, 10 \mathrm{~min} ., 37^{\circ} \mathrm{C}\right)$ and the pellet of platelets remained on the bottom of the test tube. The platelet-poor plasma (PPP) was poured out and the pellet was diluted by $1 \mathrm{ml}$ of Tyrode's solution. The next step included the magnetic labelling and magnetic separation of the cells. We added $20 \mu \mathrm{L}$ of CD45 MicroBeads and 20 $\mu \mathrm{L}$ of CD235a MicroBeads into the sample and gently mixed. After incubation we filled the column with the sample of labelled cells to obtain the leukocyte and erythrocyte depleted sample. The number of cells in the final platelet samples was determined by the blood cell analyser.

On the basis of previous experience we decided to change some conditions again for our further experiments. The first change was that we immediately added prostaglandin E1 (PGE1) into the samples at the start of the experiment. We tried to prevent the platelet 
activation at all points. The buffer was also changed and the pellet was subsequently diluted by Tris buffer solution. The pure platelet samples were obtained from 50 patients by methods which were described previously. Before further analyses, the samples have been stored with RNAlater ${ }^{\circledR}$ Tissue Collection: RNA Stabilization Solution (Life Technologies Corporation, Carlsbad, USA) at $-80{ }^{\circ} \mathrm{C}$. To confirm our results we performed cytometry analyses of the final platelet samples (labelling of the cells with CD42b antibody) and miRNA isolation from pure platelets using High Pure miRNA Isolation Kit (F. Hoffmann-La Roche Ltd, Basel Switzerland) according to manufacturer's instructions. From $150 \mu \mathrm{l}$ of thawed platelet samples 50ul of miRNA was obtained with a concentration about $1-2 \mathrm{ng} / \mathrm{ml}$. The isolation step was controlled using RNA Spike-in (UniSP2, 4 and 5 RNA) (QIAGEN GmbH, Hilden, Germany). Pilot miRNA Assays were performed using miRCURY LNA miRNA PCR Starter Kit (QIAGEN GmbH, Hilden, Germany) which normally contains hsa-miR-103a-3p PCR Assay and two LNA PCR Assays of the choice (hsa-miR-126-3p and has-miR-145-5p). The RNA spike-in control assay of cDNA synthesis targets the UniSp6 RNA. Final PCR assays were performed in duplexes. Because of a low concentration of isolated miRNAs the manufacturer protocol was modified, cDNA was diluted 1:10 for following PCR assays.

\section{RESULTS}

We prepared 50 pure platelet samples (leukocyte-depleted and erythrocyte-depleted platelet samples; WBC: $0.00 \times 10^{9} / 1$; RBC: $0.00-0.01 \times 10^{9} / 1$; PLT: $36-288 \times 10^{9} / 1$ values using blood cell analyser) (Tab.1) which will be used for further genetic analysis. Using flow cytometry analyses we confirmed the high purity of the platelet samples after the

Table 1: Example of initial and final cell counts of the samples measured by the blood cell analyser

\begin{tabular}{|l|c|c|c|c|c|c|}
\hline & $\begin{array}{c}\text { WBCs } \\
\left(10^{9} / 1\right)\end{array}$ & $\begin{array}{c}\text { WBCs a.s. } \\
\left(10^{9} / 1\right)\end{array}$ & $\begin{array}{c}\text { RBCs } \\
\left(10^{12} / 1\right)\end{array}$ & $\begin{array}{c}\text { RBCs a.s. } \\
\left(10^{12} / 1\right)\end{array}$ & $\begin{array}{c}\text { PLTs } \\
\left(10^{9} / 1\right)\end{array}$ & $\begin{array}{c}\text { PLTs a.s. } \\
\left(10^{9} / 1\right)\end{array}$ \\
\hline LCD concentrate sample & 0.00 & 0.00 & 0.01 & 0.01 & 1307 & 180 \\
\hline Patient 1 & 0.2 & 0.00 & 0.01 & 0.01 & 543 & 261 \\
\hline Patient 2 & 0.1 & 0.00 & 0.01 & 0.00 & 444 & 218 \\
\hline Patient 3 & 0.1 & 0.00 & 0.01 & 0.00 & 503 & 243 \\
\hline Patient 4 & 0.00 & 0.00 & 0.01 & 0.00 & 149 & 110 \\
\hline Patient 5 & 0.1 & 0.00 & 0.01 & 0.00 & 554 & 265 \\
\hline Patient 6 & - & 0.00 & - & 0.00 & - & 173 \\
\hline Patient 7 & - & 0.0 & - & 0.00 & - & 200 \\
\hline Patient 8 & - & 0.00 & - & 0.00 & - & 211 \\
\hline Patient 9 & - & 0.00 & - & 0.00 & - & 195 \\
\hline Patient 10 & - & 0.00 & - & 0.00 & - & 233 \\
\hline
\end{tabular}

Abbr.: LCD: Leucocyte-depleted platelet, WBCs: white blood cells, RBCs: red blood cells, PLTs: platelets, a.s.: after separation 
separation as we found that only a minor part (0.053\%-0.081\%) of the cells in the platelet samples after separation were not determined as platelets (the amount of 100,000 measured cells) (Fig.2). We also measured the platelet activation in the platelet samples after the separation by means of the CD62P expression using flow cytometry analyses. In concordance with our hypothesis the platelet activation measured by the CD62P expression after the platelet separation was lower in the samples with PGE1 compared to the samples without PGE1(Fig.3). The LNA PCR Assays gave consistent results with Cq values (PCR cycle number at which fluorescence reaction curve intersects the threshold line) between 17 and 33 .
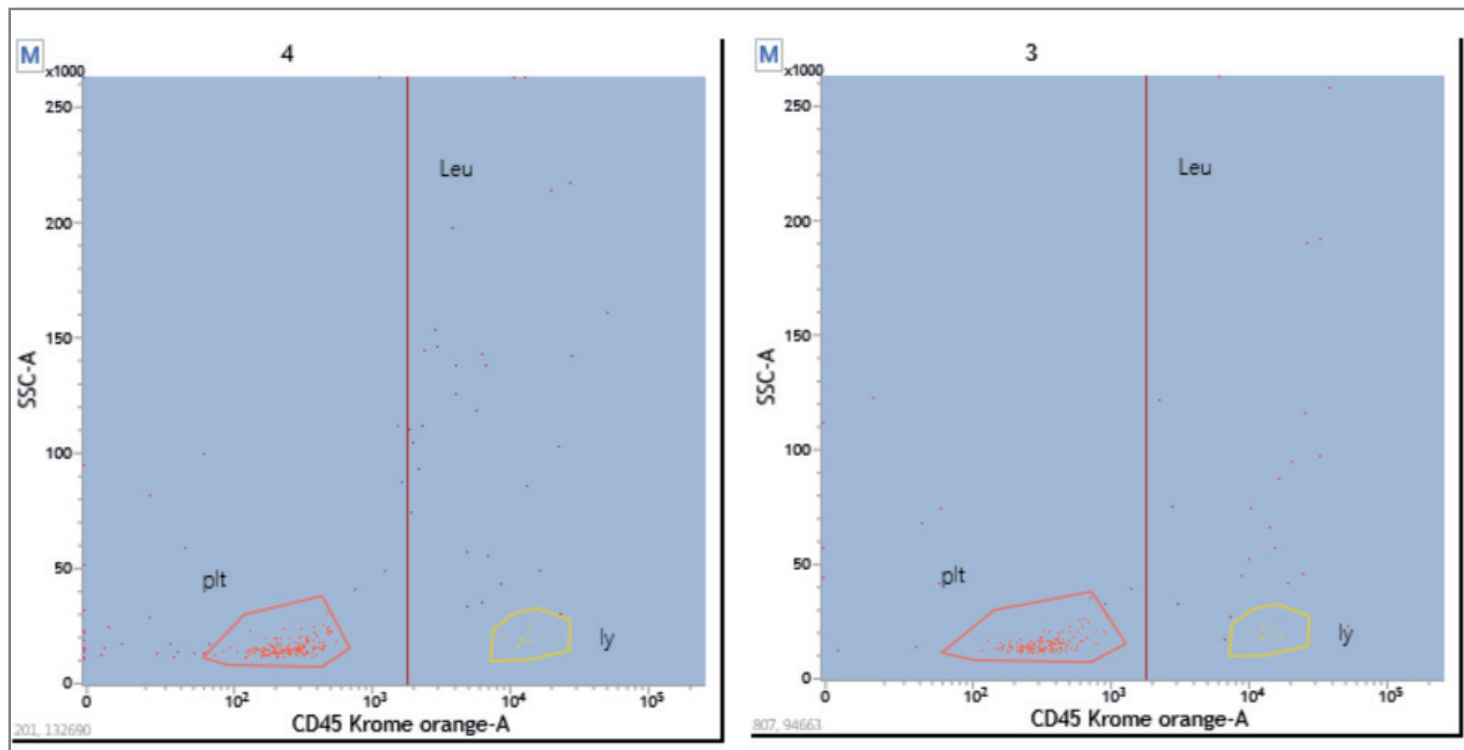

Fig. 2. Results from flow cytometry analyses confirmed the purity of samples.

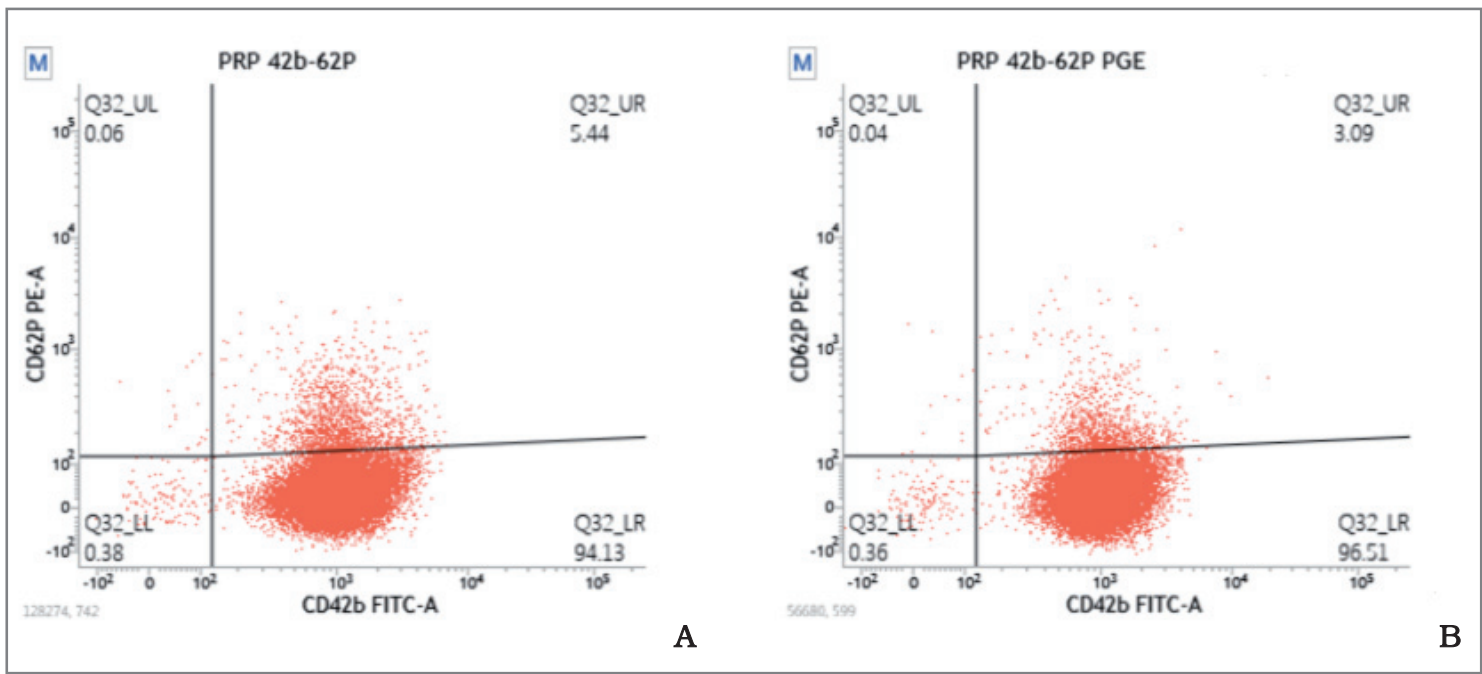

Fig. 3. Results from flow cytometry analyses confirmed the lower platelet activation after PGE1 addition. (A: sample without PGE1, B: sample with PGE1 addition.) 


\section{DISCUSSION}

Our attempts to optimize the method for the platelet separation can be considered as effective and reproducible. Described experiments have several limitations, so we had to change some conditions. In our first experiment we found that there was an extremely low count of platelets in the final sample compared to the platelet concentrate at the beginning. We thought that this low amount of platelets was due to the platelets damage by centrifugation and, subsequently, nonspecific labelling to leucocytes and erythrocytes. After elution of the labelled cells the results from blood cell analyser showed that there was a higher platelet count than in the first fraction. Therefore, we changed centrifugation conditions and labelling time. After this change the results from the blood cell analyser (Tab.1) showed that we obtained leukocyte-depleted and erythrocyte-depleted platelet samples. However, our other attempts were unsuccessful, thus we had to change also other conditions. We used PGE 1 to prevent the platelet activation, and Tris buffer solutions for diluting. After this modification we successfully repeatedly obtained leukocytes and erythrocytes depleted platelet samples. It is very important to manipulate with platelets samples gently, use only plastic tools. It is also very important to secure the best depletion of leukocytes and erythrocytes. Leukocytes contain about thousands fold more RNA and it could subsequently lead to contamination of the platelet samples [15].

There are a lot of variations in this method. Discussion can be about various filters, using only CD45 MicroBeads, or skip of adding PGE1, different time of centrifugation, and many others. Study group of Kondkar et al. used protocol by Weyrich laboratory with the CD45 positive cell depletion [9]. Another study of Nagalla et al. also used a separation method with the CD45 MicroBeads, and this protocol yield a purity of less than 1 leukocyte per 5 million platelets [15]. We additionally used the CD235a MicroBeads to ensure the purity from erythrocytes. Gnatenko et al. used the gel filtration and nylon filament filter [16], but we decided to skip that because of the excessive manipulation with platelets and their possible further activation. Also the blood volume is an often changed parameter in the cross-study comparisons, or it is not defining. Variation in the measurement platforms may cause differences in results, so lack of the universally optimized measurement platforms and lack of the guidelines is a big challenge in the platelet RNA research.

MiRNA has been the most studied small RNAs, but comparatively a little is known about the role of miRNA in platelets. For future, platelet miRNA has the potential to become a suitable biomarker of various diseases, including SPS. Another option is to use platelet miRNA as a therapeutic agent in the form of antiplatelet drugs, as well as its use in the early diagnostics of diseases. Many studies have shown the importance of miRNA not only in hematological diseases. The development of new technologies that would provide the conditions for faster extraction and direct analysis of platelet miRNA should contribute to the diagnostics of various diseases, including SPS, and also to a better understanding of the mechanisms of the genes in the platelets. Results from pilot miRNA assays are very promising, now our goal will be to determine the set of miRNAs differentially expressed in platelets of patients with SPS. Our attempts of platelets separation using the magnetic separation system seem to be the first in Slovakia. However, according to our opinion it is necessary to improve the platelet separation techniques and other methods associated with laboratory work on platelets and standardize them.

\section{REFERENCES}

1. Sokol J, Skerenova M, Jedinakova Z, et al. Progress in the Understanding of Sticky Platelet Syndrome. Seminars in Thrombosis and Hemostasis 2016; 43(1):8-13.

2. Sunderland N, Skroblin P, Barwari T, et al. MicroRNA Biomarkers and Platelet Reactivity. The Clot Thickens. Circ Res 2017;120(2):418-435. 
3. Kubisz P. Response to Comment on Sticky platelet syndrome. Semin Thromb Hemost., 2014;40 (2):274.

4. Ferreiro JL, Gómez-Hospital JA, Angiolillo DJ. Platelet abnormalities in diabetes mellitus. Diab Vasc Dis Res 2010;7(4):251-259.

5. Bergmeier W, Wagner DD. Inflammation. In: Michelson AD, ed. Platelets. 2nd ed. Oxford, United Kingdom: Elsevier Inc; 2007:117-144.

6. Santimone I, Gemmati D. Sticky Platelet Syndrome and the Role of Glycoprotein Receptors: A Review of Literature. J Blood Disorders Transf 2013; 4:6.

7. Lee RC, Feinbaum RL, Ambros V. The C. elegans heterochronic gene lin-4 encodes small RNAs with antisense complementarity to lin-14. Cell 1993;75: 843-854.

8. Landry P, Plante I, Ouellet DL, et al. Existence of a microRNA pathway in anucleate platelets. Nat Struct Mol Biol 2009; 16:961-966.

9. Kondkar AA, Bray MS, Leal SM, et al. VAMP8/endobrevin is overexpressed in hyperreactive human platelets: suggested role for platelet microRNA. J Thromb Haemost 2010 ;8(2):369-78.

10. Graham GJ, Ren Q, Dilks JR, et al. Endobrevin/VAMP-8-dependent dense granule release mediates thrombus formation in vivo. Blood 2009;114(5):1083-90.

11. Elgheznawy A, Fleming I. Platelet-Enriched MicroRNAs and Cardiovascular Homeostasis. Antioxid Redox Signal $2018 ; 29(9): 902-921$.

12. Kaudewitz D, Skroblin P, Bender LH, et al. Association of MicroRNAs and YRNAs With Platelet Function. Circ Res $2016 ; 118(3): 420-432$.

13. Mammen EF. Ten years' experience with the "Sticky platelet syndrome”. Clin Appl Thromb Hemost 1995; 1:66-72.

14. Bick RL. Sticky platelet syndrome: A common cause of unexplained arterial and venous thrombosis. Clin Appl Thromb Hemost 1998; 4:77-81.

15. Nagalla S., Shaw Ch., Kong X. et al. Platelet microRNA-mRNA coexpression profiles correlate with platelet reactivity. Blood 2011; 117: 5189-5197.

16. Gnatenko D.V., Dunn J.J., McCorkle S.R., et al. Transcript profiling of human platelets using microarray and serial analysis of gene expression. Blood. 2003 Mar 15;101 (6):2285-93.

17. Bartošová L., Fedorová J., Ivanková J., et al. Sticky platelet syndrome: where is its cause? Acta Medica Martiniana. 2008; 8(3): 23-29.

Online:

https://www.miltenyibiotec.com/_Resources/Persistent/e7a31d4b47c2c1caa28502c65c10a346f12f 3bd/30238_Illustration_positive_selection_direct_labeling-1170x475.png

Acknowledgments: This work was supported by grants VEGA 1/0187/17, VEGA 1/0168/16 and cofinanced by EU project CEPV II (ITMS 26220120036) and APVV 16-0020.

Received: March, 7, 2019

Accepted: September, 4, 2019 